\title{
Are central nervous system drugs displaying anti-inflammatory activity suitable for early treatment of COVID-19?
}

\author{
Paweł Grieb ${ }^{1}$, Konrad Rejdak ${ }^{2}$ \\ ${ }^{1}$ Mossakowski Medical Research Institute, Polish Academy of Sciences, Warsaw, Poland, ${ }^{2}$ Department of Neurology, \\ Medical University of Lublin, Lublin, Poland
}

\begin{abstract}
The majority of COVID-19 cases are only mildly or moderately symptomatic, but in some patients excessive inflammatory response becomes the dominant factor of disease progression to the advanced stage, with high mortality. Treatment with anti-inflammatory drugs either does not prevent disease progression (non-steroidal anti-inflammatory drugs [NSAIDs], colchicine), or is recommended only at the advanced disease stage (dexamethasone). Fluvoxamine and amantadine are drugs used to treat neurological and psychiatric diseases. Fluvoxamine is a selective serotonin uptake inhibitor, whereas amantadine is an old antiviral variably influencing brain neurotransmitter systems, and repurposed to Parkinson's disease. Both drugs are agonists of sigma-1 receptors located in the endoplasmic reticulum, which effect seems responsible for their anti-inflammatory activity. Moreover, amantadine was found to dampen the expression of cathepsin-L, a lysosomal enzyme implicated in SARS-CoV-2 virus entry to target cells. In two small controlled clinical trials, early treatment of SARS-CoV-2-infected persons with fluvoxamine fully prevented COVID-19 symptoms. Anecdotal evidence shows that amantadine may be similarly effective. Both drugs are easily available, inexpensive and have favorable safety profiles. Clinical trials evaluating their efficacy as much-needed post-exposure prophylaxis and early treatment of COVID-19 are ongoing.
\end{abstract}

Key words: fluvoxamine, amantadine for COVID-19.

\section{Introduction}

In the National Institutes of Health (NIH) Covid-19 Treatment Guidelines [39], it has been remarked that a large volume of data and publications of various quality pertinent to the treatment of COVID-19 are emerging at a very rapid pace. Some are published in highly respected peer-reviewed medical journals, others as preprints, and others as press releases. The available data are being continuously reviewed, assessed for scientific rigor and validity, and ultimately incorporated into guidelines to which medical practitioners should adhere. Use of untested and unproven therapies is not recommended and should be discouraged. Such practice should be universally accepted across the world. On the other hand, in the aforementioned Treatment Guidelines it is admitted that "the choice of what to do or not to

Communicating author:

Prof. Paweł Grieb, Mossakowski Medical Research Institute, Polish Academy of Sciences, 5 Pawińskiego St., 02-106 Warsaw, Poland, e-mail: pgrieb@imdik.pan.pl 
do for an individual patient is ultimately decided by the patient and their provider".

A major problem in the therapy of COVID-19 is that the vast majority of high quality trials were focused on treating hospitalized patients, and patients are hospitalized when the disease reaches the advanced stage. A recent systematic literature review on COVID-19 therapies [58] presents results of trials in which the effects of antivirals, antimalarial and mucolytic drugs, etc. on duration of hospitalisation of COVID-19 patients were assessed. It would be difficult to find a better illustration for the paucity of attempts of early, pre-hospital treatment of COVID-19.

In a highly cited paper presenting an in-depth analysis of the transcriptional response to SARSCoV-2 and its comparison with responses to other respiratory viruses [5] the authors indicated a unique and inappropriate inflammatory response to the virus as the major defining and driving feature of COVID-19. A compelling question is at which stage of the disease this inappropriate inflammatory response is initiated. Does it start only when the disease matures and overt signs of lung inflammation become detectable? Or, in particular in patients with risk factors such as various chronic diseases and/or old age, is the inappropriate inflammatory response initiated already in the early, pre-hospital phase of the disease? Should presymptomatic or early symptomatic COVID-19 patients not be treated early with anti-inflammatory drugs in order to avoid possible development of inappropriate inflammatory response? Are there not several well-established, inexpensive drugs which could be used to prevent the development of a malicious COVID-19 inflammation?

For counteracting fever and pain in the pre-hospital COVID-19 phase paracetamol is frequently recommended, despite it displays a minor anti-inflammatory activity and may deplete cellular glutathione [50]. Early use of non-steroidal anti-inflammatory drugs (NSAIDs) such as ibuprofen could be more advisable [30], although the common knowledge is that the effects of these highly popular drugs are not impressive in this disease.

Dexamethasone, a corticosteroid prescribed in a wide range of conditions for its anti-inflammatory and immunosuppressant effects, has been shown to diminish, but not abolish mortality when given to COVID-19 hospitalized patients suffering from pneu- monia and oxygen-dependent [32,44], and such treatment is currently recommended. Dexamethasone has not been tested in early disease in consideration that its immune-suppressive effects could worsen clinical outcomes in this setting [31]. A more promising drug for early treatment of COVID-19 seemed to be colchicine, an old drug frequently used in auto-immune and inflammatory diseases [37], but patients taking colchicine were found not to be protected against severe COVID-19 [6,20].

Two drugs currently known primarily for their activity on the central nervous system (CNS), fluvoxamine and amantadine, gained considerable attention recently as possible post-exposure prophylaxis and early treatment of COVID-19. In either case the reason is that anti-inflammatory activity overlaps direct action on CNS. For each of these drugs a brief outline of pharmacology and justification for a possible repurposing to the early treatment of COVID-19 is presented below.

\section{Fluvoxamine pharmacology}

This drug belongs to selective serotonin reuptake inhibitors (SSRI) facilitating serotoninergic neurotransmission by selectively inhibiting serotonin $(5 \mathrm{HT})$ reuptake at the presynaptic membrane. It is extensively used for treatment of depression and anxiety disorders. Although less potent towards $5 \mathrm{HT}$ receptors than some other SSRIs (e.g., paroxetine, sertraline, citalopram), fluvoxamine differs from the other drugs of this class in that it has virtually no or much less affinity for other receptors in the brain (e.g. muscarinic, histaminergic, etc.) [59]. A notable exception is a distinct subtype of opioid receptors resistant to classical opioid antagonists, naloxone and naltrexone, known as sigma-1 receptors (S1R), to which fluvoxamine is the agonist most active among all SSRI drugs [28].

Antidepressants belonging to the SSRI exert potent anti-inflammatory effects in the brain. An example is attenuation of brain cytokine expression in experimental stroke by fluoxetine [34]. Attenuation of intracerebral inflammation is considered an important aspect of the anti-depressive efficacy of these drugs [19]. A mechanism of the anti-inflammatory effects of SSRIs is related to the aforementioned sigma-1 receptor. This unique receptor resides in the membranes of the endoplasmic reticulum (ER), in particular at the interface between 


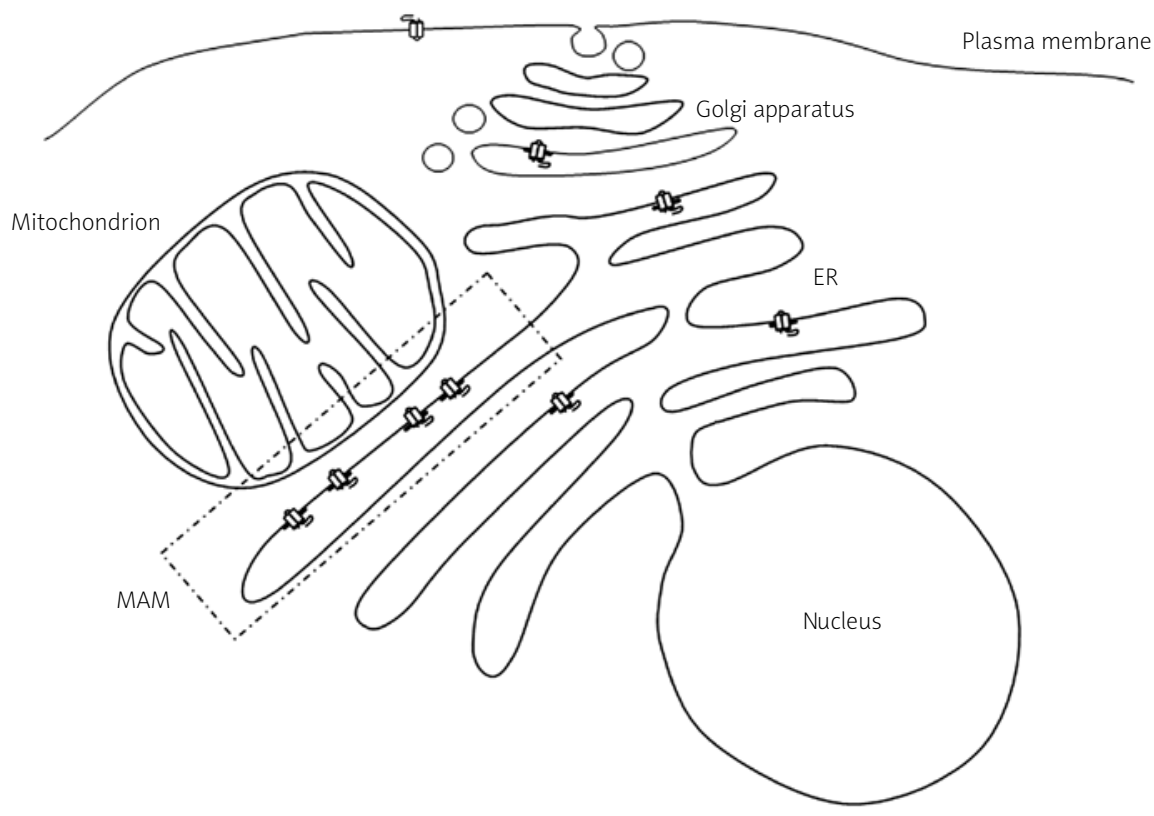

Fig. 1. Scheme illustrating location of transmembrane protein known as the sigma-1 receptor in various parts of the cellular membrane system. MAM, mitochondrion-associated membrane, a region of the endoplasmic reticulum tethered to mitochondria.

ER and mitochondria called mitochondrion-associated membrane (MAM), and is able to translocate to the plasma membrane as well, as to the nuclear envelope. Such subcellular localization enables this transmembrane protein to regulate communication between mitochondria, nucleus and cell outer membrane [53] (Fig. 1). One of the major functions of S1R is chaperoning proteins passing through this structure and acting as a pluripotent modulator of their function. S1Rs agonists, by increasing chaperoning the target proteins in ER, exert anti-inflammatory activity in the brain by counteracting endoplasmic reticulum stress, which effect is implicated in the neuroprotective activity of these drugs [48].

\section{Fluvoxamine for COVID-19}

Some SSRIs, e.g. fluoxetine, exert antiviral activity toward a broad spectrum of viruses including HIV-1, Ebola, Coxsackie virus, and others (reviewed in [43]). In the context of COVID-19 even more important could be effects of these drugs on the immune system, because serotonin acts as an immunomodulator downregulating inflammatory response by both central and peripheral mechanisms [14]. Importantly, it has been argued that when COVID-19 disease progresses toward a severe form, disease mecha- nisms related directly to the viral infection become less and less important and disease drivers become more and more related to excessive and inadequate inflammatory responses [22,51].

As already mentioned, fluvoxamine is unique among SSRIs because, besides serotonergic receptors, it does not interact with other receptors, with the exception of the sigma-1 receptor. S1R is abundant in many brain regions, but is also present in several peripheral organs, such as the lung, heart, liver, and others. Acting via these receptors fluvoxamine exerts anti-inflammatory activity also in the periphery, e.g. in the intestine [2] and heart [23]. Rosen et al. [47] discovered that the drug protects mice from lethal septic shock provoked by lipopolysaccharide and dampens the inflammatory response in human blood leukocytes, and suggested that it may prove useful for sepsis control.

In a placebo-controlled outpatient study, Lenze et al. [33] discovered that fluvoxamine given as early treatment for mild COVID-19 illness totally prevents disease progression. In that study 152 adult COVID-19 patients were randomized to fluvoxamine or placebo starting within 7 days from symptom onset. During subsequent 2 weeks, clinical deterioration occurred in 6 of 72 patients taking placebo, 


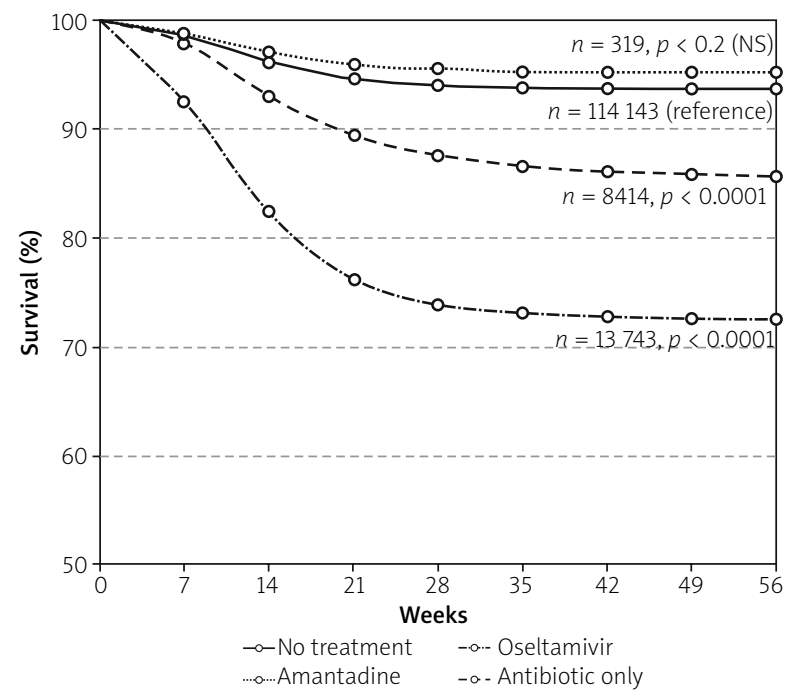

Fig. 2. Short-term survival of Mexico City patients infected with SARS-CoV-2 depending on treatment with antivirals or antibiotics. The fraction of survivors was assessed at weekly intervals. The graph complied using the data selected from those in Figure $2 \mathrm{~d}$ in ref. [25]. According to this source, $P$ is the probability (calculated using Logrank Mantel-Cox pairwise comparison) that survival of treated patients differs from survival of the reference group which received no antiviral or antibiotic.

but in none of patients taking fluvoxamine. In the other study [49], 113 patients infected with SARSCoV-2 virus were offered fluvoxamine, of which 65 were taking the medicine whereas 48 refused such treatment. Two weeks later none of the patients who took fluvoxamine had disease symptoms, while in the no-treatment group, $60 \%$ were symptomatic and $12.5 \%$ required hospitalization.

Both aforementioned clinical studies were inspired by the observation that fluvoxamine antiinflammatory effect appears to be mediated by agonism with S1R. However, involvement of other mechanisms, such as reduction of histamine release from mast cells, lysosomotropic effects of the drug interfering with viral trafficking, and the others, is also considered [54]. One interesting explanation of the apparent beneficial effect of fluvoxamine in COVID-19 [3] is related to inhibition of melatonin-metabolizing liver cytochrome leading to a substantial increase in the plasma level of this substance [57]. Melatonin, a neurohormone produced mainly by the pineal gland and cells of the gastrointestinal tract, is known to modulate several physiological functions, including activities of the immune system [24]. Almost 10 years ago it has been hypothesized that melatonin acts as a kind of "immune buffer" exerting immunostimulatory effects under immunosuppressive conditions, but acting as an anti-inflammatory compound in acute inflammation [11]. More recently a potential of melatonin for controlling inflammatory responses to COVID-19 has been suggested [46].

An extensive post-marketing surveillance based on data of 17 years of global use and estimated 28 million patients exposed to the drug confirmed the favourable safety profile of fluvoxamine [8], although a more recent analysis [52] indicated danger from interaction with warfarin and other anticoagulants. Clinical trials of fluvoxamine in COVID-19 are currently on-going in Hungary (NCT04718480), the USA (NCT04668950) and Brazil (NCT04727424).

\section{Amantadine pharmacology}

This drug effectively blocked the $M 2$ viroporin channel of the influenza A virus, and was the first antiviral approved by the US Food and Drug Administration (FDA) for prophylaxis against Asian influenza in 1966. In 2009, amantadine (together with its derivative rimantadine) was proposed for the inclusion in the World Health Organisation (WHO) Model List of Essential Medicines for the prophylaxis and treatment of influenza A, specifically the subtype H5N1 known of its high case fatality rate [1]. Unfortunately, soon after that influenza viruses developed resistance to these drugs [27] and amantadine is no longer recommended in this setting, although some manufacturers did not remove influenza $A$ as the indication for the drug. However, it should be noted that amantadine may interact with viroporins and display antiviral activity toward RNA viruses other than influenza A, such as Chikungunya virus [16]. A particularly interesting case is a positive result of a recently published randomized, placebo-controlled trial in which amantadine was used as an active treatment of Borna virus-infected depressive patients [17], although it is not clear whether the positive outcome of this trial was related to amantadine antiviral activity, to its antidepressant effect, or a mixture of both.

Currently amantadine is used mainly as a neurological drug. It was formally repurposed to Parkinson's disease, and is also used off-label in traumatic brain injury (TBI), multiple sclerosis, etc. Amanta- 
dine displays good penetrance to CNS and variably influences different neurotransmitter systems (dopaminergic, adrenergic, glutamatergic) [34]. In Parkinson's disease the drug is applied to alleviate L-DOPA-induced dyskinesia, but it also exerts neuroprotection by reducing the release of pro-inflammatory factors from activated microglia and increasing the expression of glial cell line-derived neurotrophic factor (GDNF) in astrocytes [41]. The other mechanism of potential importance for neuroprotective effects of amantadine is its action as a weak glutamate/N-methyl-D-aspartate receptor antagonist that concomitantly activates the cystine/glutamate antiporter and stimulates glutathione synthesis [38]

In a recent comprehensive review [15], the most important mechanisms of amantadine pharmacological actions, identified on the basis of the in vitro and animal experiments, were compiled and related to concentrations achievable in humans. Besides the aforementioned sigma-1 receptors, the following targets for drug action are indicated: aromatic amino acid decarboxylase (the enzyme responsible for dopamine synthesis); nicotinic receptors ( $\alpha 4 \beta 2$ and $\alpha 7$ ); phosphodiesterase-1.

\section{Amantadine for COVID-19}

Amantadine belongs to adamantanes, drugs containing tricyclo-bridged hydrocarbon structure which interferes with viroporin protein channel responsible for release of RNA-viruses, such as SARS coronavirus, from infected cells [56]. It seemed possible that adamantanes could be also repurposed for COVID-19 [55].

The evidence for amantadine efficacy in COVID-19 is sparse. The first clinical observation suggestive of that this drug may alleviate development of symptomatic SARS-CoV-2 infection was a questionnaire-based study concerning a small group of patients suffering from chronic neurological diseases (Parkinson's disease and multiple sclerosis); in these patients infection with the virus was confirmed by rtPCR of nasopharyngeal swabs, but symptomatic COVID-19 disease did not develop [45]. Initially this phenomenon was interpreted as result of a possible antiviral activity of the drug, as it was suggested by the other authors [4]. Later a contribution of other mechanisms, namely anti-fatigue and pro-arousal effects of amantadine, was proposed [21].

Few case reports and small observational studies seemed to confirm the potential benefit of amantadine given as post-exposure prophylaxis or early treatment of COVID-19 [13]. A UK Biobank study [60] reported that none of the PD patients receiving this drug developed severe complications from COVID-19, and this result is in line with the hypothesis that amantadine may exert a protective effect against both COVID-19 infection and mortality. Of note is also a report from Mexico City [36], a realworld observational study in which survival curves of patients with laboratory-confirmed COVID-19 treated with particular antiviral and/or antibiotic medicine were compared to the survival curve of the group receiving no treatment. The data, an excerpt of which is shown in Figure 2, seem rather strange as it is evident that mortality of patients treated with antimicrobial drugs (e.g. oseltamivir, antibiotics) was significantly higher than mortality of untreated patients. A likely explanation is that these drugs were applied to more severely sick patients and were ineffective. However, the survival curve of amantadine-treated patients was not significantly different from the survival curve of patients receiving either antivirals or antibiotics. Whereas this observation cannot be taken as a sign of amantadine efficacy in COVID-19 (perhaps amantadine was offered to less sick patients), it may be interpreted as a proof that treatment with this drug is safe in the COVID-19 setting.

In Poland, despite the lack of a formal proof of efficacy, treatment of COVID-19 with amantadine has been advocated in media, advertised by some physicians (see e.g. [26]), and became considerably popular. Rumours spread about fast and dramatic subjective improvements encountered by patients infected with SARS-CoV-2 and taking this drug. Subjective improvements could result from anti-fatigue and pro-arousal effects of amantadine, which were mentioned above.

A classic test of the antiviral activity of a drug is based on the assessment of the effect on proliferation of viruses in a green African monkey kidney cell line Vero. In this experimental setup, amantadine inhibited SARS-CoV-2 replication with $\mathrm{IC}_{50}$ concentrations of 83 to $119 \mu \mathrm{M}$ [18]. After systemic administration in humans, maximal amantadine levels are about $14.6 \mu \mathrm{M}$ [7], therefore antiviral effects of the drug should not be expected. However, it may interfere with the development of a severe COVID-19 through mechanisms interfering with the development of uncontrolled inflammation, not detectable using the in vitro test on Vero cells. First, the drug 
at concentrations easily achievable, following intake of therapeutic doses is a potent agonist of S1R [15], thus in COVID-19 it may, similarly to fluvoxamine, counteract the development of uncontrolled inflammatory reaction.

The second important mechanism of amantadine's therapeutic activity in COVID-19 may involve cathepsin $L$ (CatL). This lysosomal enzyme is one of the proteases known to participate in the entry of SARS-CoV-2 to cells. Coronaviruses use spike glycoprotein (S) to bind their receptor, mediate membrane fusion and virus entry to target cells. Depending on virus strain and cell type, coronaviral S protein may be cleaved by one or several host proteases. A study by Ou et al. [42] indicated that CatL is engaged in the entry to cells equipped with human angiotensin converting enzyme 2 (hACE2) receptor. Importantly, in human lungs, CatL is expressed in bronchial epithelial cells and macrophages [9].

Zhao et al. [61] provided evidence for the crucial role of CatL in patients with COVID-19. These authors found that the level of this protein in the circulating blood was significantly higher in COVID-19 patients than in the healthy volunteers. The plasma levels of CatL were markedly higher in patients with severe disease compared to those with milder disease. Further, the elevated level of CatL was found in SARS-CoV-2 pseudovirus-infected cells in vitro. Moreover, when the authors investigated the effects of amantadine, they found that the drug inhibited CatL enzyme activity in cells subjected to SARS-CoV-2 pseudovirus infection and significantly inhibited SARS-CoV-2 entry to the cells, with little cytotoxicity. Finally, in the humanized mice infected with the pseudovirus amantadine decreased CatL expression. The authors concluded that amantadine may be a potent therapeutic drug for COVID-19, able to halt a vicious cycle in which enhancement of CatL expression triggered by viral infection takes place.

The aforementioned mechanisms could, indeed, explain alleged improvement of patients suffering from COVID-19 following amantadine intake. Needless to say, a prospective, controlled study is necessary to confirm such effect. Currently a placebo-controlled trial to assess amantadine efficacy for preventing progression of early COVID-19 toward pulmonary and hyper-inflammatory phase has been initiated in Poland (NCT04854759), and a similar trial is being set up in Denmark (NCT04894617).

\section{Conclusions and future prospects}

Fluvoxamine and amantadine are currently not established as antivirals. If they prove efficacious in the treatment of SARS-CoV-2-infected patients, they might rather be classified as host-directed therapies (HDT), i.e. drugs that target host factors instead of the virus directly. HDT approach is well known in medicine, although it is used mostly for treatment of chronic diseases, e.g. chronic viral hepatitis [29]. An advantage of such strategy is that mutations of the virus would not influence drug efficacy.

On the other hand, there is a caveat. A recent in vitro and murine study [12] demonstrated that commonly used NSAIDs, ibuprofen and meloxicam, had no effect on viral entry and replication, but reduced the pro-inflammatory cytokine response to SARS-CoV-2 infection as well, as the antibody response. Such effects could prevent the development of immunity toward the virus. Drugs exerting anti-inflammatory effects, even if proven effective for early COVID-19, should not become an alternative for the vaccination against SARS-CoV-2.

\section{Disclosure}

The authors report no conflict of interest.

\section{References}

1. $17^{\text {th }}$ Expert Committee on the Selection and Use of Essential Medicines. Geneva, March 2009. Proposal for the inclusion of amantadine in the World Health Organisation Model List of Essential Medicines. pdf4pro.com/view/proposal-for-the-inclusion-of-amantadine-8db6d.html (accessed June 23, 2019).

2. Almási N, Török S, Valkusz Z, Tajti M, Csonka Á, Murlasits Z, Pósa A, Varga C, Kupai K. Sigma-1 receptor engages an anti-inflammatory and antioxidant feedback loop mediated by peroxiredoxin in experimental colitis. Antioxidants (Basel) 2020; 9: 1081.

3. Anderson GM. Fluvoxamine, melatonin and COVID-19. Psychopharmacology (Berl) 2021; 238: 611.

4. Araújo R, Aranda-Martínez JD, Aranda-Abreu GE. Amantadine treatment for people with COVID-19. Arch Med Res 2020; 51: 739-740.

5. Blanco-Melo D, Nilsson-Payant BE, Liu WC, Uhl S, Hoagland D, Møller R, Jordan TX, Oishi K, Panis M, Sachs D, Wang TT, Schwartz RE, Lim JK, Albrecht RA, tenOever BR. Imbalanced host response to SARS-CoV-2 drives development of COVID-19. Cell 2020; 181: 1036-1045.e9.

6. Bourguiba R, Delplanque M, Vinit C, Ackermann F, Savey L, Grateau G, Hentgen V, Georgin-Lavialle S. Clinical course of COVID-19 in a cohort of 342 familial Mediterranean fever patients with a long-term treatment by colchicine in a French endemic area. Ann Rheum Dis 2020; annrheumdis-2020-218707. 
7. Brenner M, Haass A, Jacobi P, Schimrigk K. Amantadine sulphate in treating Parkinson's disease: clinical effects, psychometric tests and serum concentrations. J Neurol 1989; 236: 153-156.

8. Buchberger R, Wagner W. Fluvoxamine: safety profile in extensive post-marketing surveillance. Pharmacopsychiatry 2002; 35: 101-108.

9. Bühling F, Waldburg N, Reisenauer A, Heimburg A, Golpon H, Welte T. Lysosomal cysteine proteases in the lung: role in protein processing and immunoregulation. Eur Respir J 2004; 23: 620-628.

10. Butterworth RF. Potential for the repurposing of adamantane antivirals for COVID-19. Drugs R D. 2021; Jun 21.

11. Carrillo-Vico A, Lardone PJ, Alvarez-Sánchez N, RodríguezRodríguez A, Guerrero JM. Melatonin: buffering the immune system. Int J Mol Sci 2013; 14: 8638-8683.

12. Chen JS, Alfajaro MM, Chow RD, Wei J, Filler RB, Eisenbarth SC, Wilen CB. Non-steroidal anti-inflammatory drugs dampen the cytokine and antibody response to SARS-CoV-2 infection. J Virol 2021; 95: e00014-21.

13. Cortés-Borra A, Aranda-Abreu GE. Amantadine in the prevention of clinical symptoms caused by SARS-CoV-2. Pharmacol Rep 2021; 73: 962-965.

14. Costa LHA, Santos BM, Branco LGS. Can selective serotonin reuptake inhibitors have a neuroprotective effect during COVID-19? Eur J Pharmacol 2020; 889: 173629.

15. Danysz W, Dekundy A, Scheschonka A, Riederer P. Amantadine: reappraisal of the timeless diamond-target updates and nov el therapeutic potentials. J Neural Transm (Vienna) 2021; 128 127-169.

16. Dey D, Siddiqui SI, Mamidi P, Ghosh S, Kumar CS, Chattopadhyay S, Ghosh S, Banerjee $M$. The effect of amantadine on an ion channel protein from Chikungunya virus. PLoS Negl Trop Dis 2019; 13: e0007548.

17. Dietrich DE, Bode L, Spannhuth CW, Hecker $H$, Ludwig $H$, Emrich HM. Antiviral treatment perspective against Borna disease virus 1 infection in major depression: a double-blind placebo-controlled randomized clinical trial. BMC Pharmacol Toxicol 2020; 21: 12

18. Fink $K$, Nitsche A, Neumann M, Grossegesse $M$, Eisele $K H$, Danysz W. Amantadine Inhibits SARS-CoV-2 in vitro. Viruses 2021; 13: 539

19. Gałecki P, Mossakowska-Wójcik J, Talarowska M. The anti-inflammatory mechanism of antidepressants - SSRIs, SNRIs. Prog Neuropsychopharmacol Biol Psychiatry 2018; 80: 291-294.

20. Gendelman O, Amital H, Bragazzi NL, Watad A, Chodick G. Continuous hydroxychloroquine or colchicine therapy does not prevent infection with SARS-CoV-2: Insights from a large healthcare database analysis. Autoimmun Rev 2020; 19: 102566.

21. Grieb P, Świątkiewicz M, Prus K, Rejdak K. Amantadine for COVID-19. J Clin Pharmacol 2021; 61: 412-413.

22. Grieb P, Świątkiewicz M, Prus K, Rejdak K. Hypoxia may be a determinative factor in COVID-19 progression. Curr Res Pharm Drug Discovery 2021; 2: 100030.

23. Guo Y, Zhang C, Chen X, Liu X, Ye T, Fo Y, Shi S, Qu C, Liang J, Shen B, Yang B. Sigma-1 receptor ligands improves ventricular repolarization-related ion remodeling in rats with major depression disorder. Psychopharmacology (Berl) 2021; 238: 487-499.
24. Hardeland R, Cardinali DP, Srinivasan V, Spence DW, Brown GM, Pandi-Perumal SR. Melatonin - a pleiotropic, orchestrating regulator molecule. Prog Neurobiol 2011; 93: 350-384.

25. Hayashi T. The sigma-1 receptor in cellular stress signaling. Front Neurosci 2019; 13: 733.

26. https://przychodnia-przemysl.pl/how-to-treat-covid-19-in-48hours-scheme/ (accessed April 21, 2021).

27. Hussain M, Galvin HD, Haw TY, Nutsford AN, Husain M. Drug resistance in influenza $A$ virus: the epidemiology and management. Infect Drug Resist 2017; 10: 121-134.

28. Ishikawa M, Ishiwata K, Ishii K, Kimura Y, Sakata M, Naganawa M, Oda K, Miyatake R, Fujisaki M, Shimizu E, Shirayama Y, Iyo M, Hashimoto K. High occupancy of sigma-1 receptors in the human brain after single oral administration of fluvoxamine: a positron emission tomography study using [11C]SA4503. Biol Psychiatry 2007; 62: 878-883.

29. Kaufmann SHE, Dorhoi A, Hotchkiss RS, Bartenschlager R. Host-directed therapies for bacterial and viral infections. Nat Rev Drug Discov 2018; 17: 35-56.

30. Kelleni MT. Early use of non-steroidal anti-inflammatory drugs in COVID-19 might reverse pathogenesis, prevent complications and improve clinical outcomes. Biomed Pharmacother 2021; 133: 110982

31. Kim PS, Read SW, Fauci AS. Therapy for early COVID-19: A critical need. JAMA 2020; 324: 2149-2150.

32. Lee HW, Park J, Lee JK, Park TY, Heo EY. The effect of the timing of dexamethasone administration in patients with COVID-19 pneumonia. Tuberc Respir Dis (Seoul) 2021.

33. Lenze EJ, Mattar C, Zorumski CF, Stevens A, Schweiger J, Nicol GE, Miller JP, Yang L, Yingling M, Avidan MS, Reiersen AM. Fluvoxamine vs placebo and clinical deterioration in outpatients with symptomatic COVID-19: A randomized clinical trial. JAMA 2020; 324: 2292-2300.

34. Lim CM, Kim SW, Park JY, Kim C, Yoon SH, Lee JK. Fluoxetine affords robust neuroprotection in the postischemic brain via its anti-inflammatory effect. J Neurosci Res 2009; 87: 1037-1045.

35. Ma HM, Zafonte RD. Amantadine and memantine: a comprehensive review for acquired brain injury. Brain Inj 2020; 34: 299-315.

36. Mancilla-Galindo J, García-Méndez JÓ, Márquez-Sánchez J, Reyes-Casarrubias RE, Aguirre-Aguilar E, Rocha-González HI, Kammar-García A. All-cause mortality among patients treated with repurposed antivirals and antibiotics for COVID-19 in Mexico City: A real-world observational study. EXCLI J 2021; 20: 199-222.

37. Manenti L, Maggiore U, Fiaccadori E, Meschi T, Antoni AD, Nouvenne A, Ticinesi A, Cerundolo N, Prati B, Delsante M, Gandoflini I, Donghi L, Gentile M, Farina MT, Oliva V, Zambrano C, Regolisti G, Palmisano A, Caminiti C, Cocchi E, Ferrari C, Riella LV, Cravedi P, Peruzzi L. Reduced mortality in COVID-19 patients treated with colchicine: Results from a retrospective, observational study. PLoS One 2021; 16: e0248276.

38. Nakano T, Hasegawa T, Suzuki D, Motomura E, Okada M. Amantadine combines astroglial system Xc- activation with glutamate/NMDA receptor inhibition. Biomolecules 2019; 9: 191.

39. NIH COVID-19 Treatment Guidelines. https://www.covid19treatmentguidelines.nih.gov/introduction/ (accessed April 21, 2021). 
40. Nisar T, Sutherland-Foggio H, Husar W. Antiviral amantadine. Lancet Neurol 2019; 18: 1080.

41. Ossola B, Schendzielorz N, Chen SH, Bird GS, Tuominen RK, Männistö PT, Hong JS. Amantadine protects dopamine neurons by a dual action: reducing activation of microglia and inducing expression of GDNF in astroglia [corrected]. Neuropharmacology 2011; 61: 574-582.

42. Ou X, Liu Y, Lei X, Li P, Mi D, Ren L, Guo L, Guo R, Chen T, Hu J, Xiang Z, Mu Z, Chen X, Chen J, Hu K, Jin Q, Wang J, Qian Z. Characterization of spike glycoprotein of SARS-CoV-2 on virus entry and its immune cross-reactivity with SARS-CoV. Nat Commun 2020; 11: 1620.

43. Pashaei Y. Drug repurposing of selective serotonin reuptake inhibitors: Could these drugs help fight COVID-19 and save lives? J Clin Neurosci 2021; 88: 163-172.

44. RECOVERY Collaborative Group, Horby P, Lim WS, Emberson JR, Mafham M, Bell JL, Linsell L, Staplin N, Brightling C, Ustianowski A, Elmahi E, Prudon B, Green C, Felton T, Chadwick D, Rege K, Fegan C, Chappell LC, Faust SN, Jaki T, Jeffery K, Montgomery A, Rowan K, Juszczak E, Baillie JK, Haynes R, Landray MJ. Dexamethasone in hospitalized patients with Covid-19. N Engl J Med 2021; 384: 693-704.

45. Rejdak K, Grieb P. Adamantanes might be protective from COVID-19 in patients with neurological diseases: multiple sclerosis, parkinsonism and cognitive impairment. Mult Scler Relat Disord 2020; 42: 102163.

46. Reynolds JL, Dubocovich ML. Melatonin multifaceted pharma cological actions on melatonin receptors converging to abrogate COVID-19. J Pineal Res 2021; e12732.

47. Rosen DA, Seki SM, Fernández-Castañeda A, Beiter RM, Eccles JD, Woodfolk JA, Gaultier A. Modulation of the sigma-1 receptor-IRE1 pathway is beneficial in preclinical models of inflammation and sepsis. Sci Transl Med 2019; 11: eaau5266.

48. Ruscher K, Wieloch T. The involvement of the sigma-1 receptor in neurodegeneration and neurorestoration. J Pharmacol Sci 2015; 127: 30-35.

49. Seftel D, Boulware DR. Prospective cohort of fluvoxamine for early treatment of coronavirus disease 19. Open Forum Infect Dis 2021; 8: ofab050.

50. Sestili P, Fimognari C. Paracetamol-induced glutathione consumption: Is there a link with severe COVID-19 illness? Front Pharmacol 2020; 11: 579944

51. Siddiqi HK, Mehra MR. COVID-19 illness in native and immunosuppressed states: A clinical-therapeutic staging proposal. J Heart Lung Transplant 2020; 39: 405-407.

52. Spina E, Barbieri MA, Cicala G, Bruno A, de Leon J. Clinically relevant drug interactions between newer antidepressants and oral anticoagulants. Expert Opin Drug Metab Toxicol 2020; 16 31-44.

53. Su TP, Su TC, Nakamura Y, Tsai SY. The sigma-1 receptor as a pluripotent modulator in living systems. Trends Pharmacol Sci 2016; 37: 262-278.

54. Sukhatme VP, Reiersen AM, Vayttaden SJ, Sukhatme VV. Fluvoxamine: A Review of Its Mechanism of Action and Its Role in COVID-19. Front Pharmacol 2021; 12: 652688.

55. Tipton PW, Wszolek ZK. What can Parkinson's disease teach us about COVID-19? Neurol Neurochir Pol 2020; 54: 204-206.
56. Torres J, Maheswari U, Parthasarathy K, Ng L, Liu DX, Gong X. Conductance and amantadine binding of a pore formed by a lysine-flanked transmembrane domain of SARS coronavirus envelope protein. Protein Sci 2007; 16: 2065-2071.

57. von Bahr C, Ursing C, Yasui N, Tybring G, Bertilsson L, Röjdmark $\mathrm{S}$. Fluvoxamine but not citalopram increases serum melatonin in healthy subjects - an indication that cytochrome P450 CYP1A2 and CYP2C19 hydroxylate melatonin. Eur J Clin Pharmacol 2000; 56: 123-127.

58. Welte T, Ambrose LJ, Sibbring GC, Sheikh S, Müllerová H, Sabir I. Current evidence for COVID-19 therapies: a systematic literature review. Eur Respir Rev 2021; 30: 200384.

59. Westenberg HG, Sandner C. Tolerability and safety of fluvoxamine and other antidepressants. Int J Clin Pract 2006; 60: 482-491.

60. Yu Y, Travaglio M, Popovic R, Leal NS, Martins LM. Alzheimer's and Parkinson's diseases predict different COVID-19 outcomes: A UK Biobank Study. Geriatrics (Basel) 2021; 6: 10.

61. Zhao MM, Yang WL, Yang FY, Zhang L, Huang WJ, Hou W, Fan CF, Jin RH, Feng YM, Wang YC, Yang JK. Cathepsin L plays a key role in SARS-CoV-2 infection in humans and humanized mice and is a promising target for new drug development. Signal Transduct Target Ther 2021; 6: 134. 